\title{
RISK AMONG CLEANERS: THE USAGE OF PERSONAL PROTECTIVE EQUIPMENT (PPE)
}

\author{
Nur Syazwani Mohd Nawi ${ }^{*}$, Ting Huey Yin ${ }^{2}$, Ezrin Hani Sukadarin ${ }^{3}$ \\ 1 School of Technology Management and Logistics, Universiti Utara Malaysia, Malaysia \\ Email: nursyazwani@uum.edu.my \\ 2 School of Technology Management and Logistics, Universiti Utara Malaysia, Malaysia \\ Email: ting_huey@stml.uum.edu.my \\ 3 Faculty of Industrial Sciences and Technology, Universiti Malaysia Pahang, Malaysia \\ Email: ezrin@ump.edu.my \\ * Corresponding Author
}

\section{Article Info:}

Article history:

Received date: 03.03.2020

Revised date: 27.04 .2020

Accepted date: 10.05 .2020

Published date: 10.06 .2020

\section{To cite this document:}

Nawi, N.S. M., Ting, H. Y., \& Sukadarin, E. H. (2020). Risk among Cleaners: Risk among Cleaners: The Usage of Personal Protective Equipment (PPE). Journal of Information System and Technology Management, 5 (17), 01-11.

DOI: $10.35631 /$ JISTM.517001.

\begin{abstract}
:
Personal Protective Equipment (PPE) is involved in many industries including cleaning nowadays. The influence factors and usage levels of PPE are very different based on groups. The data in this research is to collect and analysis through the qualitative method. A total of 34 cleaners was observed and interviewed. In this study, the influence factors of PPE usage included the scope of job tasks and works environment, organization culture, rule and enforcement, PPE accessibility/ availability, personal feeling and perspective, knowledge and awareness on safety and hazard factors, and personal behavior. The result also showed that the PPE usage condition is good at a moderate level which fits the cleaning tasks and works environment. The risk of cleaners is low. However, UCLEANSB takes safety action to maintain and enhance the PPE usage among cleaners. For further study, the study can implement to other diverse cleaning group samples with different job task categories.
\end{abstract}

Keywords:

Personal Protective Equipment, Usage, Risk, Cleaners

\section{Introduction}

Every job has its own risks, even cleaning also no exception. Based on the study of Ilesanmi and Omotoso (2015), the work of a cleaning may have a great impact on cleaners' body especially among female cleaners (Alamgir \& Yu, 2008; Perkarinen, 2009; Matulonga et al., 2016). Cleaners more easily exposed to more hazardous risk environment. For example, the extensive use of chemical detergents, the specification of detergents in certain use or cleaning surface increases the need of many type detergents, the volatile of chemicals and the increased dust condition in cleaning road environment. The impact of chemicals and detergents skin contact, inhalation of chemicals volatile and dust, splash of liquid or gas detergents to eyes and 
other hazard factors to human body may not notice and detect by cleaners from the beginning, but the effect and injury such as organs damage, respiratory system problem (asthma), visual problem and other health problem that caused by it will show out after long-term contacts, like the truth of dripping water wears away a stone (Matulonga et al., 2016). Therefore, to provide a protection to employees, a personal protective equipment (PPE) is designed and introduced.

In fact, PPE should apply in all work task to provide protection to all categories of employees. The use of PPE basically come after engineering controls and administrate controls in ergonomics stressors control strategies. Personal protective equipment now is common use by employees in job tasks and operation process. It is one of the strategies for an employer to provide welfare for employees by protecting employees form hazard factors. However, there is some problem will face by employers in the effort to reduce employees hazard risk exposure with PPE implementation (Dolev \& Vu-Khanh, 2009). The previous study by Ilesanmi et al (2015), Tam \& Fung (2008) and MacFarlane et al (2008) showed that although PPE is prepared by an employer to employees, most of them still not fully use the equipment prepared. There are also have a research prove that the different job tasks will reason a different level of PPE usage (Fischer, Hynes, \& Perl, 2014). The usage level between different type of job task and employees' categories is uncertainty. It is important to identify the usage level of PPE among employees before thinking a new strategy, whether the application of PPE need to improve or not for more effectively on hazard factors reduction and employee's protection.

In the other hand, a factor that influences the use of PPE among employees is different based on different internal and external factors. Based on previous research of DellaValle et al (2012), the decision to use PPE may be based on several factors including social norms, perceived effectiveness, pesticide-related health issues experiences, safety training, cost and risk perception and others. Besides, the research of Tam \& Fung (2008) showed another influencing factor of using PPE. To ensure the effectiveness of employee's hazards factors reduction effort, it is better to identify the specific influence factors of the target employees group.

This study is conducted with the objective to identify the type of PPE that use by cleaners in their job task with examine the PPE usage level at the same time and also study the influence factors in using PPE among them. Identify the level of PPE usage among cleaners is the basic to find the problem and solution on hazard factors control and then this study will be able to improve cleaners' health and reduce the risk of hazard factors exposure.

\section{Literature Review}

\section{Personal Protective Equipment (PPE)}

Personal protective equipment (PPE) is a tool or equipment that designed to protect human from serious workplace injury or health problem that cause by the contact and exposure to workplace hazard factors (U.S. Department of Health \& Human Services, 2017). It is a human protective strategy that needs a person to wear something to protect themselves and avoid contact with harmful factors. Personal protective equipment may include items such as gloves, safety goggles, safety shoes, ear plugs, safety helmets, respiratory devices, vests, body clothing as showed in Figure 1, and more based on the categories of ears, feet, hands and arms, body, airways and protective clothing (DellaValle et al., 2012). It protects human from chemicals, radioactive, physical, electrical, mechanical and other hazard factors to reduce the risk of injury and dangerous (Perry et al., 2009). 


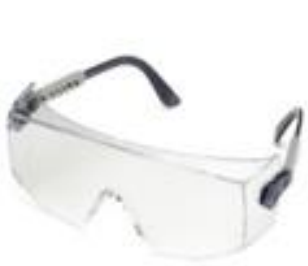

(a)

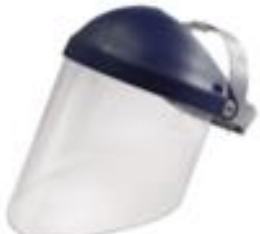

(b)

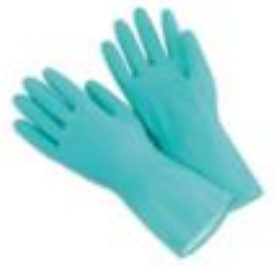

(c)

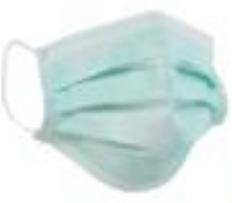

(d)

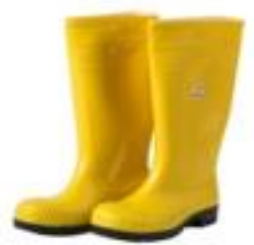

(e)

Figure 1: Example of Personal Protective Equipment (PPE) (a) Safety glasses (b) Face shield (c) Gloves (d) Mask (e) Safety boots

Personal protective equipment is one of the workplace hazard and stressor control strategies in ergonomics. The use of personal protective equipment is usually essential but compared with other stressors control in the hierarchy of ergonomics controls, PPE is arranged at the bottom, which means that it is usually the last line of defense after other control strategies (Floyd, 2015; Jensen, 2014). Based on the hierarchy of control in ergonomics stressor control that showed in Figure 2, PPE is applying as the last defense line in hazard control after other hazards control because of its effectiveness and business value. The research of Ilesanmi et al. (2015) stated that PPE can only reduce the risk of injury if used consistently and correct ways, but not eliminate it. In fact, the more useful and worn of PPE by employees can be reduced around $50 \%$ of injury and lost work time (Lombardi et al., 2009)

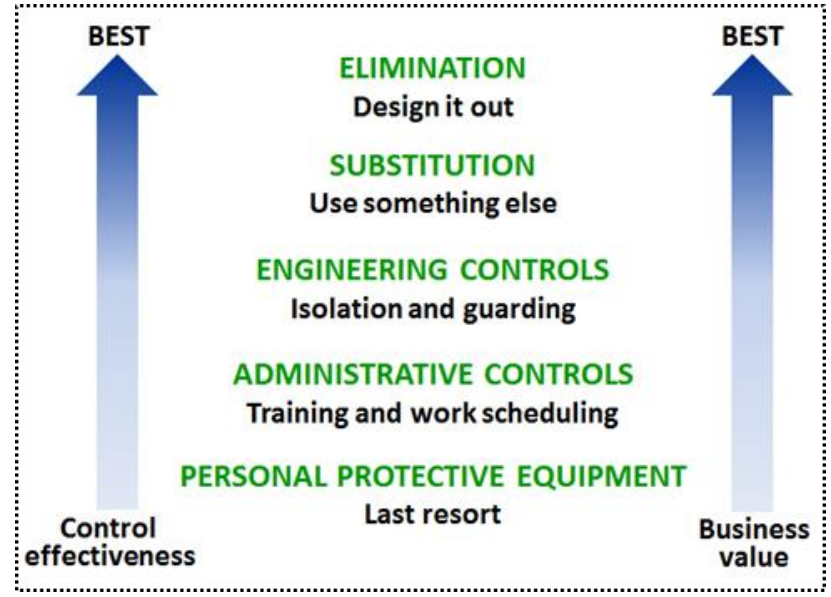

Figure 2: Hierarchy of Control in Ergonomics Stressor Control

\section{Influence Factors in using PPE}

Influence factors is an aspect or elements that may give effect or impact on employee's decision. The use of PPE can be influenced by internal factors or external factors. Based on the previous research of DellaValle et al. (2012), the decision to use PPE is influenced by some external factor like social norms, chemical-related health issues experiences, safety training, cost and risk perception and so on. In the other hand, the past research of Tam \& Fung (2008) showed the use of PPE is affected by personal factors like the awareness to hazard risk, recognition of risk factors, feeling uncomfortable, a burden to their motion and movement and etc. Moreover, the acceptance, flexibility, compatibility with ongoing work and other more subtle issues also may affect the use of PPE as stated in the result of MacFarlane et al. (2008). 
Okello et al. (2017) have another result showed that the issue of PPE training, availability and access, promotion and enforcement and knowledge on PPE purpose also will affect the PPE usage by employees. While a study of Ravichandran \& Brundha (2016) showed the colleagues recommendation on PPE and PPE that good quality and cheap are significant factors that considered by employees. Everyone is different with their experiment, knowledge, and behavior. All the result proves that the influence factors in using PPE are various and uncertainty based on the user.

After analyzed from previous studies, influence factors can be summarized into seven main categories based on external and internal factors as shown in Figure 3. External factors included the organization culture, rule and enforcement, scope of job tasks and work environment, and accessibility/availability. While internal factors are more to personal factors which are knowledge and awareness of safety and hazard factors, personal feeling and perspective, and behaviors. and categorized influence factors.

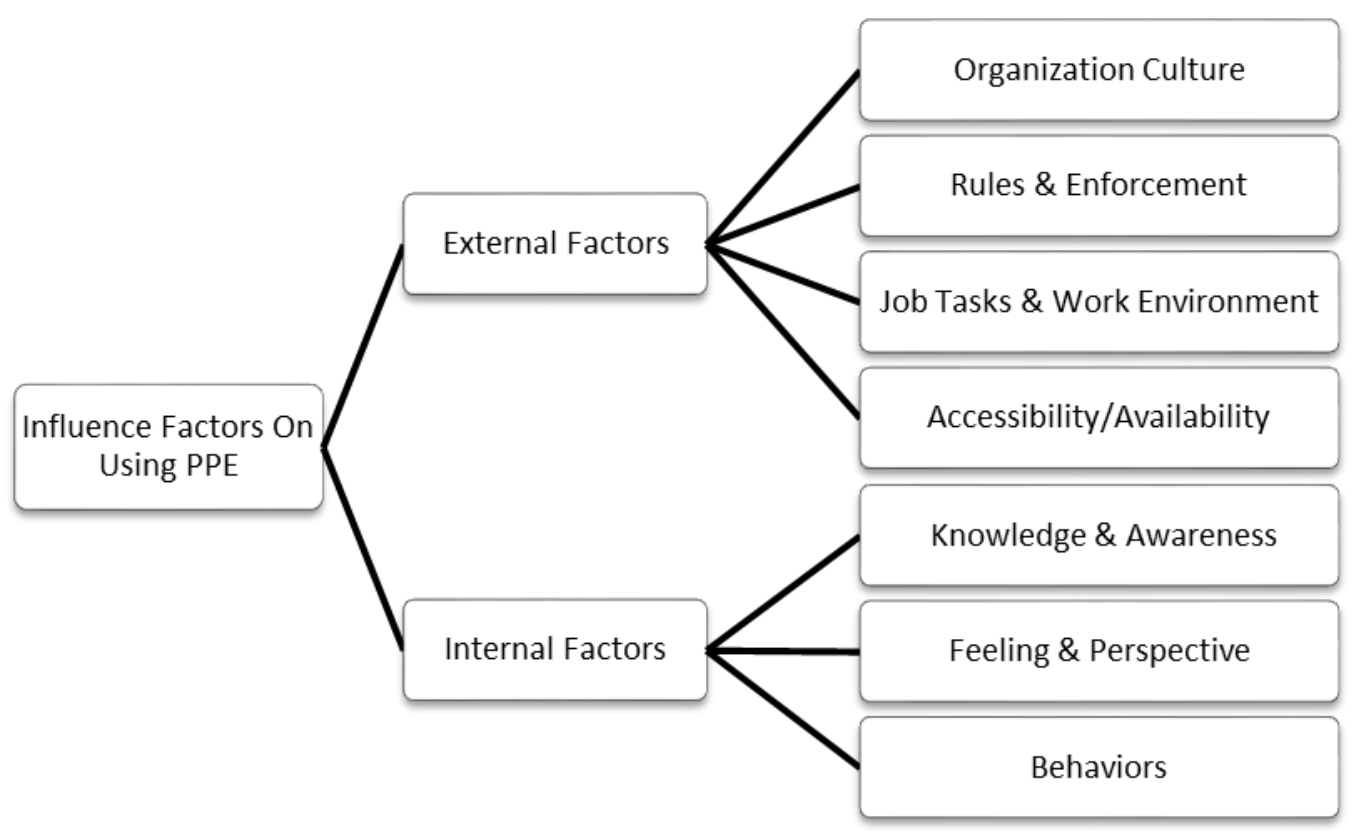

Figure 3: Categorized Influence Factors On Using PPE

\section{PPE Usage Level}

An equipment usage level is how frequent a tool or equipment is often used by users. The usage level of equipment can be measured and determine from the analysis and calculation by the record and observe the equipment using time and period of a person in a certain timeframe and situation. It shows how frequent and how many times the equipment is used and when the equipment is needed. In the other hand, it also can provide a detail data on what will cause a person using the equipment and on what situation, like the outcome from the research of DellaValle et al (2012) and Braham, Finch \& McIntosh (2004). A PPE usage level in different job tasks or industry and employees group or categories is different as shown in the previous research result of Ilesanmi et al. (2015), Tam \& Fung (2008) and MacFarlane et al (2008). In addition, the study result of MacFarlane, Chapman, Benke et al. (2008) and Fischer et al. (2014) stated the PPE usage level among farmers is low and PPE usage level among medicine employees is high respectively. However, the study of Okello et al. in 2017 stated up the situation of low PPE usage among cleaners and nurses. Even if in the high-risk industry such as construction, the PPE usage also low because of the uncomfortable and low awareness of 
the risk factors (Tanko \& Anigbogu, 2016). This situation showed that although PPE is essential for every employee in self-protection and injury prevention, the usage level of PPE is still changing depend on the group of employees themselves.

\section{Methodology}

This study was carried out among the UCLEANSB cleaners that located at students' resident's hall in university campus. UCLEANSB is a company that support and manages job relating to cleaning, indoor and outdoor maintenance, landscape maintenance and customer service complaints unit in university campus.

This study focuses on cleaners' group in accommodation based on their frequent contact rate with chemical detergent and dust factors after compared with other areas like office and academic buildings. In fact, the dirty level at accommodation areas is higher than office and academic areas, especially in the toilet and bathroom. The degree of use of accommodation also high as areas that living by students whole the day within the study period.

The data in this study was collected qualitatively which comprised of the interview to UCLEANSB cleaners and observation on their used equipment when doing job tasks. Face to face structured interview among cleaners is conducted to identify the significant influence factors in PPE usage, the usage level and type of PPE them use in dairy job tasks.

\section{Data Analysis and Discussion}

\section{Demographic Statistic}

There are 34 UCLEANSB cleaners involved in the study. Based on the data in Table 1, most of the respondent is more than 45 years old and working period with tend to less than 4 years and more than 12 years. The data have shown the number of respondents that assigned to job tasks have distinct based on gender. Most of the female respondents involved in all type of job tasks like toilet and bathroom cleaning, corridor cleaning, street and road cleaning, and clean up trash. In other hands, male respondents are less be responsible for the toilet and bathroom cleaning and corridor tasks because most of the students' accommodation is living by female students. Cleaners also involved in the extra tasks that only carry out when university semester break period, accommodation room cleaning, and ditch sterilization and antisepsis.

Table 1: Respondents Demographic Statistic (Total N = 34)

\begin{tabular}{lcccc}
\hline & \multicolumn{4}{c}{ Gender } \\
\cline { 2 - 5 } & Frequency & $\begin{array}{c}\text { Percent } \\
\%\end{array}$ & Frequency & $\begin{array}{c}\text { Percent } \\
\%\end{array}$ \\
\cline { 2 - 5 } & & & & \\
\hline Age & 0 & $0.0 \%$ & 0 & $0.0 \%$ \\
$\quad$ 17-25 years old & 5 & $14.7 \%$ & 4 & $11.8 \%$ \\
26-35 years old & 3 & $8.8 \%$ & 1 & $2.9 \%$ \\
36-45 years old & 11 & $32.4 \%$ & 10 & $29.4 \%$ \\
$\quad$ >45 years old & & & & \\
\hline Working Period With & & & & $8.8 \%$ \\
UCLEANSB & 7 & $20.6 \%$ & 3 & $2.9 \%$ \\
$\quad$ <4 year & 3 & $8.8 \%$ & 1 & \\
5-8 year & & & & \\
\hline
\end{tabular}




\begin{tabular}{|c|c|c|c|c|}
\hline $\begin{array}{l}9-12 \text { year } \\
>12 \text { vear }\end{array}$ & $\begin{array}{l}3 \\
6\end{array}$ & $\begin{array}{l}8.8 \% \\
176 \%\end{array}$ & 6 & $17.6 \%$ \\
\hline \multicolumn{5}{|c|}{ Job Scope Involved: } \\
\hline \multicolumn{4}{|c|}{ Toilet \& Bathroom } & \\
\hline Yes & 18 & $52.9 \%$ & 3 & $8.8 \%$ \\
\hline No & 1 & $2.9 \%$ & 12 & $35.3 \%$ \\
\hline \multicolumn{5}{|c|}{ Corridor Cleaning } \\
\hline Yes & 18 & $52.9 \%$ & 8 & $23.5 \%$ \\
\hline No & 1 & $2.9 \%$ & 7 & $20.6 \%$ \\
\hline \multicolumn{5}{|c|}{ Street \& Road Cleaning } \\
\hline Yes & 9 & $26.5 \%$ & 10 & $29.4 \%$ \\
\hline No & 10 & $29.4 \%$ & 5 & $14.7 \%$ \\
\hline \multicolumn{5}{|c|}{ Clean Up Trash } \\
\hline Yes & 18 & $52.9 \%$ & 10 & $29.4 \%$ \\
\hline No & 1 & $2.9 \%$ & 5 & $14.7 \%$ \\
\hline
\end{tabular}

\section{Influence Factor of PPE Usage}

Based on the data interview, the significant influence factors in using PPE is identified will come together with other factors. Except for connection between external or internal factors, the connection within the external and internal factors in PPE usage also occur as analysis at below.

\section{Job Tasks and Work Environment - Personal Perspective and Feeling}

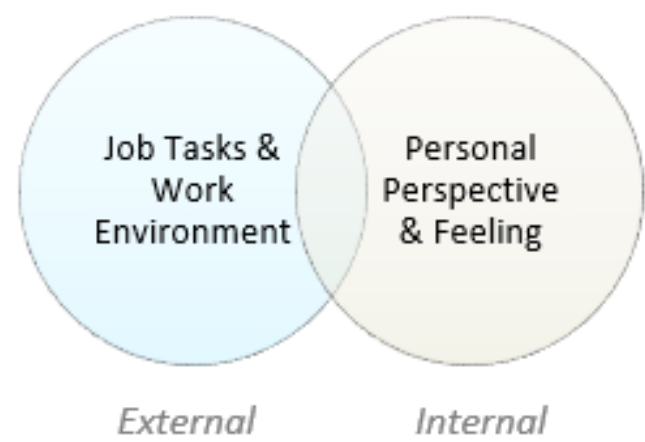

\section{Figure 4: Job Tasks \& Work Environment and Personal Perspective \& Feeling}

First, the influence of the type of job tasks and work environment together with the personal perspective and feeling on the jobs hazard. All the cleaners interviewed stated that he/she will only wear complete PPE that need when he/she are carry the job like cleaning ditches and toilet that involved the use of highly dangerous risk chemicals detergent like chlorine. Other jobs like cleaning students' accommodation bathroom, sweep the floor, cleaning the street and road, and clean up trash is considered as low hazards job task by cleaners. In their perspective, the use of high acid level detergent like chlorine (as used by all cleaners) is very dangerous and need to wear PPE like mask and glove when using. Sometimes, he/she will wear others clothing when using chlorine. In other hands, when using other neutral chemical level detergent as provided by UCLEANSB, they no feel any dangerous on it although in fact they no really know the detergent is neutral and no dangerous for human body. Most of the cleaner make own Copyright $\odot$ GLOBAL ACADEMIC EXCELLENCE (M) SDN BHD - All rights reserved 
conclusion that the cleaning detergent is not dangerous to them based on their experience in using such type of detergents.

In addition, for the job tasks that only carry out on university semester break holiday like accommodation's room and whole accommodation area cleaning, the usage level of PPE will higher than daily because they will contact with high dust level in a closed area and chemicals for a more longer time in a day.

Only several cleaners say that he/she need to wear completed PPE included mask, glove, boots, protective suite (plastic apron) to avoid the contact and splash of soap/detergent water because he/she know that he/she are already old to resisting chemical hazard effect on their body and immune system. While other job tasks like sweeping the floor, clean up trash and other that exposure in a dusk environment, most of them say that they are doing cleaning tasks at an open environment, it is ok for them to no wearing any respiratory PPE. For them who are sensitive will wear up a mask. In addition, the use of glove very depends on the current situation that occurs.

\section{Organization Culture - Rules and Enforcement by UCLEANSB}

Although the use of PPE when doing job task is requested by UCLEANSB and company rule (no stated in the contract), but it is highly affected by the organizational culture. All of the cleaners interviewed is recognized and acknowledge the importance of PPE and rules in UCLEANSB. In fact, the usage of PPE is not same as their acknowledge level. They know the necessary of PPE, but no really will follow and do it.

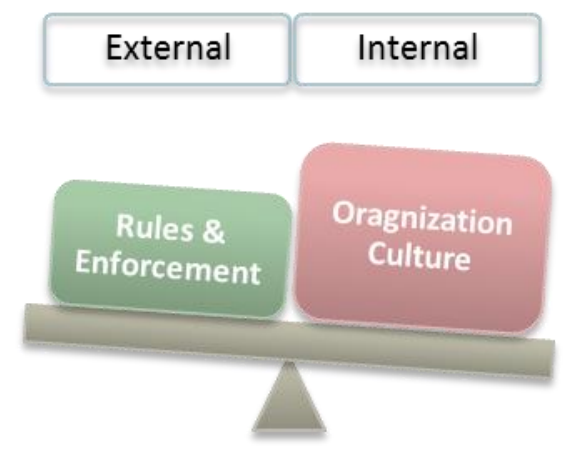

Figure 5 : Influence of Organization Culture On Rule and Enforcement

When a person first comes to the job, UCLEANSB will provide a program about job task and equipment needed. In around 200 page of power slide with a proper explanation by the supervisor before start work. All of them with know the use of PPE is compulsory. After really work at accommodation with other colleagues they will affect by colleagues behaviors and perspective. In other hands, the enforcement of using PPE by UCLEANSB is no really strict.

From the analyzed data, the influence level of organizational culture is less in small cleaners group because one of the cleaners will affect only one culture. If he/she partner is often in using PPE, he/she will have the same behaviors whether wear or no wear. In large cleaners group, although there are few different people that have different PPE usage level and perspective, many of the cleaners will follow the colleagues that low in PPE usage because of the simple in work equipment and comfortable. The organization culture reduces the influence force of rules and enforcement by UCLEANSB. 


\section{Accessibility/Availability \& Knowledge and Awareness - Personal Behaviors}

For cleaners who are willing to use PPE when doing jobs, many of them have an easy going behavior. If the PPE is provided by UCLEANSB or always accessible and available, they will feel free to wear. If the PPE is no accessible, they will just do the task without PPE. Other behaviors of cleaners are they will find out a way to get PPE. No depending on the accessibility but their knowledge and awareness. Some cleaners commented that if the type of PPE they need is no accessible and available from UCLEANSB, there will buy by themselves or try to make it using other things because they know the importance of PPE and aware their tasks risk. This makes they will find out an alternative to get a PPE they need.

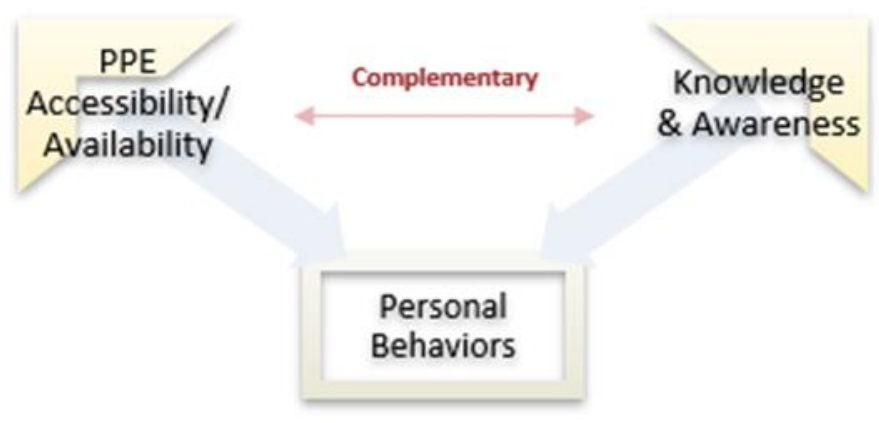

\section{Figure 6: Influence of PPE Accessibility/Availability and Knowledge \& Awareness on Personal Behaviors}

There are come to the two different responses on "would you like to use all the PPE that is required for job tasks if UCLEANSB is provided all the PPE?" First is they are willing to wear PPE that needed or extra PPE that is the request by UCLEANSB if UCLEANSB is provided all the PPE. Second is although they will wear the PPE, they still like an easy working way with less PPE.

\section{PPE Usage Level}

The data of interview on influence factors of PPE usage concluded the PPE usage level among UCLEANSB cleaners is at a moderate level and very flexible. The use of PPE very depends on the type of job tasks and cleaners' perspective to the risk of tasks. For the cleaning tasks that involved dangerous chemicals (chlorine) and detergents, the use of PPE is very frequent and always. Other the use of PPE is less and sometimes. The 3 common type of PPE that used by cleaners is gloves, mask, and boots. The type of PPE that involved in the job tasks is less, but the frequency is often. Table 2 and Figure 7 shows the PPE usage condition among UCLEANSB cleaners that estimated by each of the cleaners on he/she usage condition in daily tasks. 
Table 2 : The PPE Usage Condition of Cleaners

\begin{tabular}{|c|c|c|}
\hline Usage Condition & Frequency & Percentage (\%) \\
\hline Always & 4 & 11.8 \\
\hline $75-99 \%$ of the time & 1 & 2.9 \\
\hline $50-74 \%$ of the time & 11 & 32.4 \\
\hline $25-49 \%$ of the time & 4 & 11.8 \\
\hline $1-24 \%$ of the time & 12 & 35.3 \\
\hline Never & 2 & 5.9 \\
\hline \hline Total & $\mathbf{3 4}$ & $\mathbf{1 0 0 . 0}$ \\
\hline
\end{tabular}

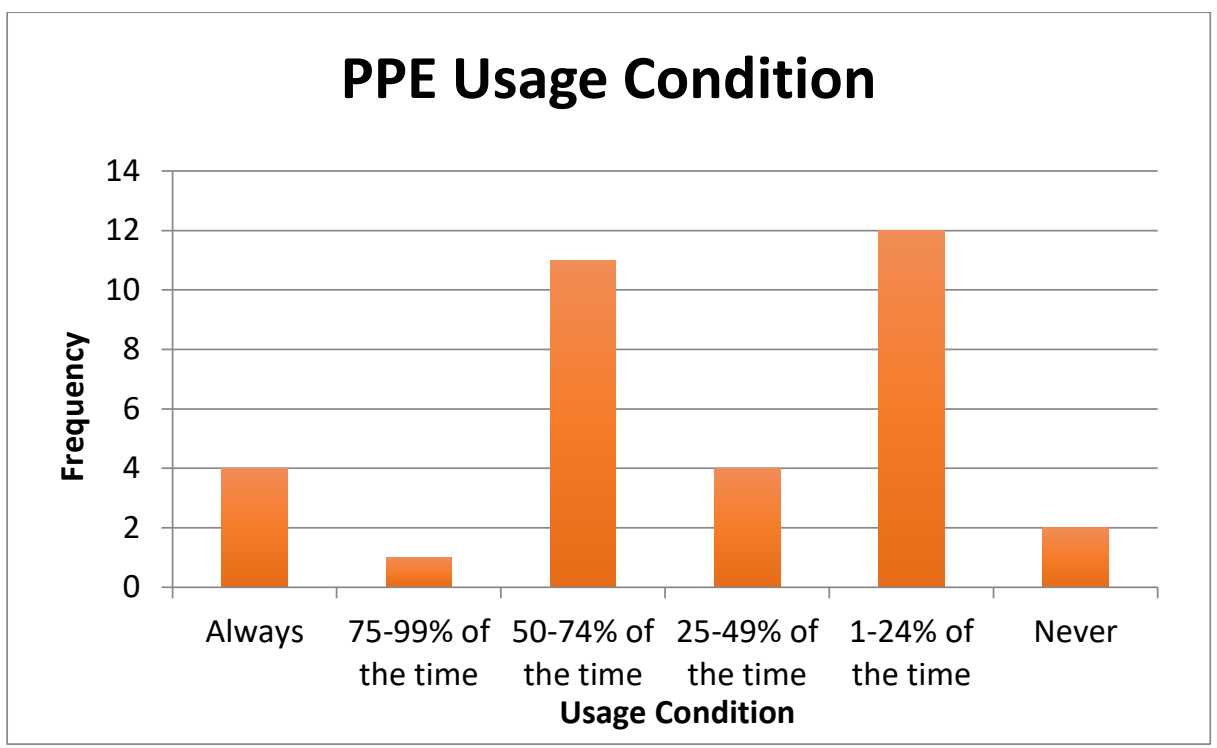

Figure 7 : The PPE Usage Condition of Cleaners

The condition of $1-24 \%$ of the time achieved the highest show most of the cleaners is carry out their task in easy mode and less wear of PPE. While around 11 cleaners are wearing PPE for $50-74 \%$ of the tasks time. The numbers of cleaners that always wearing PPE for all tasks time is same as the number of cleaners that wearing PPE 25-49\% of the tasks time. The usage condition of never and $75-99 \%$ of the time is very less at 2 and 1 cleaners respectively.

The cleaners that wearing PPE above 50\% of tasks time is more responsible to the cleaning task that involved chemical detergents, water (when cleaning bathroom) and need to clean up a branch and deciduous by using hands to avoid chemical contact, wet and scratch. In addition, the cleaners already have the habit to wear PPE when doing tasks. The cleaners that wearing PPE below 50\% are more using work tools like a broom, dustpan, and others in their tasks which enable them to direct contact with risk factors.

The observation data on cleaners daily job tasks support to the interview data outcome. The observation of PPE usage among cleaners focus on their daily job tasks situation, no included the monthly tasks ( 3 or 6 months a time). Figure 8 shows the attire and equipment that use by UCLEANSB cleaners when doing low-risk tasks such as dairy cleaning of students' accommodation bathroom, sweep the floor on accommodation area and cleaning trash. The use of PPE and condition of time in wearing PPE is fit to their low-risk tasks. 


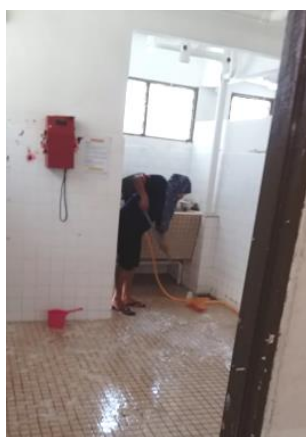

(a)

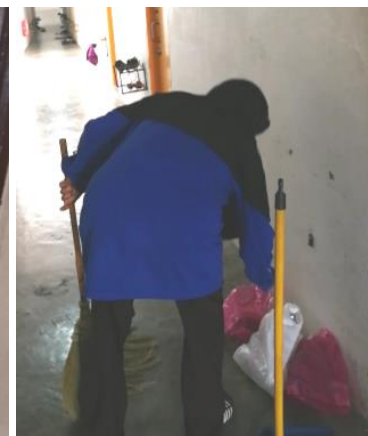

(b)

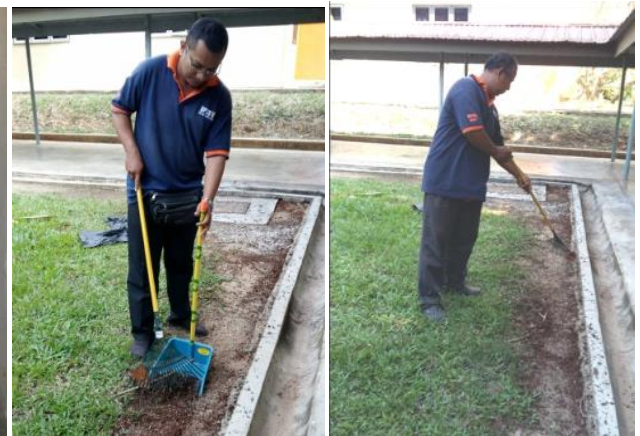

(c) (d)

Figure 8 : The Condition of UCLEANSB Cleaners in Dairy Tasks (a) Accommodation Bathroom Cleaning (b) Accommodation Corridor Sweeping (c)\&(d) Road Sweeping

\section{Conclusion}

The past studies in PPE usage factors are more to single factor analysis. However, the factors are related and able to give impact to each other. For example, the factor of personal perspective and feeling depends on the job tasks and work environment. The cleaners not only focus on their tasks requirement but based on the thinking to the tasks and work environment. The UCLEANSB cleaners achieved the moderate level in PPE usage. Most of the cleaners remain the same PPE usage condition. However, the situation of decreased PPE usage of cleaners needs to pay more attention by UCLEANSB to avoid the situation continuous increased.

The use of PPE among cleaners is in good condition. The risk of hazards in cleaning task at education areas is low compared with other industry areas. UCLEANSB have many efforts on replacing high acid and dangerous chemical detergents to neutral and low dangerous detergents to protect cleaners from chemical hazards. In fact, the case of cleaners injuries that cause by no wearing PPE in doing tasks only occur one case in the recent year. The cleaner is inhaled the chemical gas of chlorine when he/she is using chlorine without masks. Because of the case, all cleaners are aware of the dangers of chlorine and cause their will alert to use PPE when using chlorine in tasks. The function of chlorine is hard to replace by others safety chemicals, so the UCLEANSB can only give remind to cleaners to be careful in using chlorine.

There are many ways to protect cleaners from work hazards. Beside enforce the use of PPE in job tasks, replacing the dangerous chemicals with more safety chemicals also is a good strategy. It not only can save the operation cost but also improve employees comfortable when doing tasks. Factors that need UCLEANSB to pay more attention is the organizational culture which more tends to give negative impact on PPE usage among cleaners. UCLEANSB need hire external people to contribute in cleaners' PPE usage level and factors research to get more accurate and truthfulness data from cleaners.

\section{References}

Alamgir H, Y. S. (2008). Epidemiology of occupational injury among cleaners in health care section. Occupational Medicine, 58(6), 393-399.

Curt T. DellaValle, Jane A. Hoppin, Cynthia J. Hines, Gabriella Andreotti, and M., \& Alavanja, C. R. (2012). Risk-accepting Equipment, Personal Protective Equipment Use 
within the Agricultural Health Study, 17(3), 264-276. https://doi.org/10.1080/1059924X.2012.686390.Risk-Accepting

Dolev, P. I. \& Vu-Khanh, T. (2009). Recent developments and needs in materials used for personal protective equipment and their testing. International Journal of Occupational Safety and Health. 15(4), 347-362.

Fischer, W. A., Hynes, N. A., \& Perl, T. M. (2014). Protecting health care workers from Ebola: personal protective equipment is critical but is not enough. Annals of Internal Medicine, 161(10), 753-754. https://doi.org/10.7326/M14-1953

Floyd, H. L. (2015). A Practical Guide for Applying the Hierarchy of Controls to Electrical Hazards. IEEE Transactions on Industry Applications, 51(5), 4263-4266. https://doi.org/10.1109/TIA.2015.2431634

Ilesanmi OS., Omotoso B., A. I. (2015). Accidents, injuries and the use of personal protective equipment, among hospital cleaners in a tertiary hospital in south-west Nigeria., 3, 275284.

Interfaith Worker Justice, Cincinnati Interfaith Workers' Center, Houston Interfaith Worker Justice Center, A. C. W. C. (n.d.). Health And Safety In The Cleaning Services Industry-Cleaning Services Workers Training Guide.

Jensen, R. C. (2014). Hierarchies of Hazard Control: A Proposal to Resolve Different Lists. Advances in Safety Management and Human Factors, 209-216.

Lombardi, D. A., Verma, S. K., Brennan, M. J., \& Perry, M. J. (2009). Factors influencing worker use of personal protective eyewear. Accident Analysis and Prevention, 41(4), 755-762. https://doi.org/10.1016/j.aap.2009.03.017

MacFarlane E, Chapman A, Benke G, et al. (2008). Training and other predictors of personal protective equipment use in Australian grain farmers using pesticides. Occupational and Environmental Medicinea, 141-146.

Matulonga, B., Rava, M., Siroux, V., Bernard, A., Dumas, O., Pin, I., Zock, J-P., Nadif, R., Laynaert, B., Moual, N. L. (2016). Women using bleach for home cleaning are are at increased risk of non-allergic asthma. Respiratory Medicine, 117, 264-271.

Okello, T. R., Odora, J., Apio, J. A., \& Pecorella, I. (2017). Barriers and Factors Affecting Personal Protective Equipment Usage in St. Mary 's Hospital Lacor in, 22(1), 59-65.

Perkarinen A. (2009). Development in Professional Cleaning Work Brings Challenges to Ergonomics. The Ergonomics Open Journal, 2, 20-60.

R A Braham, C F Finch, A McIntosh, P. M. (2004). Community football players' attitudes towards protective equipment - a pre-season measure. Br J Sports Med, 38, 426-431. https://doi.org/10.1136/bjsm.2002.004051

Ravichandran, H., \& Brundha, M. P. (2016). Awareness about personal protective equipments in hospital workers (sweepers and cleaners) - Research. International Journal of Pharmaceutical Sciences Review and Research, 40(1), 28-29.

Tam, V. W. Y., \& Fung, I. W. H. (2008). A Study of Knowledge, Awareness, Practice and Recommendations Among Hong Kong Construction Workers on Using Personal Respiratory Protective Equipment at Risk, (61), 69-81.

Tanko, B. 1., \& Anigbogu, N. A. (2016). The Use of Personal Protective Equipment (PPE) On Construction Sites in Nigeria, (July 2012).

U.S. Department of Health \& Human Services. (2017). Personal Protective Equipment (PPE). Retrieved from https://chemm.nlm.nih.gov/ppe.htm\#clearance 